\title{
Influences of Temperature on Development and Survival, Reproduction and Growth of a Calanoid Copepod (Pseudodiaptomus dubia)
}

\author{
Changling Li, Xiaoxia Luo, Xianghu Huang*, and Binhe Gu \\ Department of Aquaculture, Fishery College, Guangdong Ocean University, \\ Xiashan, Zhanjiang, Guangdong, China \\ E-mail: hxh166@126.com
}

Received January 24, 2009; Revised July 28, 2009; Accepted July 31, 2009; Published September 1, 2009

\begin{abstract}
Pseudodiaptomus dubia is a calanoid copepod that is distributed widely in the estuarinecoastal waters of Asia and is a dominant copepod in the shrimp grow-out ponds in southern China. A laboratory culture experiment was conducted to evaluate the influences of water temperature on larval development, survival, and reproduction. Results indicate that within a temperature range from 15 to $35^{\circ} \mathrm{C}$, larval development increases as the temperature increases. The water temperature for optimal larval survival rate ranges from 20 to $35^{\circ} \mathrm{C}$. Longevity and egg hatching time decrease as the temperature increases from 20 to $35^{\circ} \mathrm{C}$. Total fecundity and reproduction frequency increase as the water temperature increases, with the maximum at $30^{\circ} \mathrm{C}$. Fecundity and reproduction frequency decrease when the temperature exceeds $30^{\circ} \mathrm{C}$. Intrinsic growth rate $\left(r_{m}\right)$ ranges from 0.168 to 0.195 at 25 to $30^{\circ} \mathrm{C}$; net reproduction rate $\left(R_{0}\right)$ and finite growth rate $(\lambda)$ are 163 to 264 and 1.183 to 1.215 , respectively, when the temperature is greater than 20 and $35^{\circ} \mathrm{C}$; population doubling time (t) varies from 3.556 to 4.128 days at temperatures less than 20 and $35^{\circ} \mathrm{C}$. Population generation time $(T)$ is negatively correlated with temperature, with the optimal population growth rate at 25 to $30^{\circ} \mathrm{C}$.
\end{abstract}

KEYWORDS: copepods, growth rate, larval development, Pseudodiaptomus dubia, temperature

\section{INTRODUCTION}

Temperature is a vital factor that influences mortality[1], instar duration[2,3], molting rate[4,5], and reproduction of copepods[5,6]. Within a suitable range, increases in temperature will increase egg production and growth rate, and shorten reproduction cycle and hatching time. Different species that live in different geographical regions may have specific requirements for temperature. Temperature affects the energy storage and hormone production that control larval development. Molting will not take place until levels of energy and hormone exceed a threshold[7].

Pseudodiaptomus dubia[8], previously reported as Schmackeria dubia[9], is a calanoid copepod that is distributed widely in Asian estuarine-coastal waters and is a dominant copepod in the shrimp grow-out 
ponds in southern China. Aside from the morphological characteristics[10], little is known about the effects of environmental factors on the ecology of $P$. dubia. Recently, we reported on the responses of feeding and filtering rates of $P$. dubia to the changes in several environmental factors, including water temperature[9]. Here we report the effects of temperature on reproduction, development, and growth. Findings from this study will provide important insights into the thermal condition that favors mass production of $P$. dubia.

\section{MATERIALS AND METHODS}

This experiment was conducted at the Guangdong Ocean University, Guangdong, China between April and June 2006. Previously, we reported the effects of several environmental variables on the filtering and feeding rates of adult $P$. dubia[9]. The objective of the current study was to assess the effects of various water temperatures on the growth and reproduction of $P$. dubia at different development stages.

\section{Experimental Animals}

P. dubia were collected from a shrimp farm (water temperature, $30^{\circ} \mathrm{C}$; salinity, $27 \mathrm{ppt}$ ) in Zhanjiang, southern China. In the laboratory, the copepods were raised at a water temperature between 28 and $30^{\circ} \mathrm{C}$, a salinity of $27 \mathrm{ppt}$, and a light intensity of 160 to $240 \mu \mathrm{mol} \mathrm{m} \mathrm{m}^{-2} \mathrm{~s}^{-1}$ [9]. After the copepods were acclimated to the culture conditions, healthy and energized egg-carrying females were carefully transferred with a pipe to the culture media. Their offspring were used as the experiment animals.

A unicellular golden alga (Isochrysis zhanjiangensis) and a diatom (Chaetoceros müelleri) provided by the Algal Culture Research Laboratory of Guangdong Ocean University (Zhanjiang, China) were used as food sources. Natural seawater was filtered using a plankton net (mesh size, $200 \mu \mathrm{m}$ ) and boiled to kill all living organisms. Wide-mouth transparent beakers $(125 \mathrm{ml})$ were used as the culture containers.

\section{Larval Selection}

Ten milliliters of fresh seawater was added to each of 200 pipettes. Copepods were transferred from the culture media and examined under a microscope. A total of 200 female copepods that were carrying matured egg sacs were then transferred into the 200 pipettes and raised at a water temperature of $30^{\circ} \mathrm{C}$, a light intensity of $200 \mu \mathrm{mol} \mathrm{m} \mathrm{m}^{-2}$, and a light:dark cycle of $12 \mathrm{~h}: 12 \mathrm{~h}$.

Newly hatched larvae were collected every $2 \mathrm{~h}$; larvae from the time period with the highest hatching rate were transferred to a beaker and used for the experiment.

\section{Experimental Design}

There were five temperature levels $\left(15,20,25,30,35^{\circ} \mathrm{C}\right)$, each of which contained 10 replicate groups. Each group within a temperature level consisted of 10 incubation bottles that were further divided into five subgroups, namely A, B, C, D, and E. Each subgroup consisted of two bottles. Each bottle had 125 $\mathrm{ml}$ seawater and 10 nauplii added to it. Group A was used to record the development and growth of nauplii and copepodites; Group B was used to record survival rate of nauplii; Group C was used to record survival rate of copepodites. Experimental animals in Groups D and E (four incubation bottles) were used for the reproduction experiment. All animals were fed with $C$. müelleri at an identical concentration of 20 $\times 10^{4}$ individuals/milliliter. Incubation was conducted at a salinity of $27 \mathrm{ppt}$, a pH of 8.0, a light intensity of $200 \mu \mathrm{mol} \mathrm{m} \mathrm{s}^{-1}$, and a light:dark cycle of $12 \mathrm{~h}: 12 \mathrm{~h}$. 


\section{Growth and Survival Rates}

The life cycle of $P$. dubia consisted of 12 stages, including six naupliar stages (NI, NII, NIII, NIV, NV, and NVI), five copepodite stages (CI, CII, CIII, CIV, and CV), and the adult stage. Because P. dubia is an egg-carrying invertebrate, eggs in the egg sacs develop into NI before the sacs are detached from the females and the NI nauplii are then hatched. NI nauplii develop into NII within 2-4 min of hatching. Therefore, our observation on larval development began at stage NII.

Developmental stages were monitored every $6 \mathrm{~h}$ by randomly taking three to four larvae from Group A bottles. The larvae were staged and returned to the same bottles. When nauplii in Group B reached NVI, they were preserved in formalin. The numbers of survival larvae and prosome length were recorded. When the larvae in Group $\mathrm{C}$ reached the adult stage, the numbers of survival and prosome length of females and males were recorded. These data were used to calculate growth and survival rates for the naupliar and copepodite stages.

Survival rate $(S R)$ was calculated using the following equation:

$$
S R=\frac{a_{1}}{10} \times 100 \%
$$

where $a_{1}$ is the live larvae at the end of the incubation. Average growth rate was calculated as

$$
\begin{aligned}
& \log C=2.00 \log C L-5.67 \text { (nauplii) } \\
& \log C=2.87 \log L-8.03 \text { (copepodites) } \\
& G=\left(C_{i+1}-C_{i}\right) / T_{i}
\end{aligned}
$$

where $C L$ and $L$ are the prosome length $(\mu \mathrm{m})$ of nauplii and copepodites, $C_{i}$ is the weight ( $\mu \mathrm{g}$ carbon) in stage $i$ larvae, and $\mathrm{T}_{i}$ is the time (days) of stage $i$ larvae.

\section{Reproductivity Experiment}

When the adult copepods in Groups D and E started carrying eggs, females and males at a ratio of 1:2 were incubated in six to 10 test tubes containing algal solutions. The hatching rate was recorded every 12 days. Incubation was not terminated until all female adults died. These data were used to calculate a reproductivity table that was then used to calculate intrinsic rate of population growth $\left(\mathrm{r}_{\mathrm{m}}\right)$, finite rate of growth $(\lambda)$, net reproduction rate $\left(R_{0}\right)$, and generation time $(\mathrm{T})$.

Life parameters of P. dubia were calculated following Shen[11] and Tang et al.[12]:

Reproduction rate:

$$
R_{0}=\Sigma l_{x} m_{x}
$$

Generation time:

$$
T=\Sigma x l_{x} m_{x} / R_{0}
$$

Intrinsic rate of population growth:

$$
r_{m}=\ln R_{0} / T
$$


Finite rate of growth:

$$
\lambda=e^{r_{m}}
$$

Population doubling time:

$$
t=(\ln 2) / r_{m}
$$

where $x$ is age or time interval, $l_{x}$ is survival rate at time $\mathrm{x}$, and $m_{x}$ is birth rate at age $\mathrm{x}$.

All statistical procedures were performed using SPSS software (version 11). Significant tests were performed using the ANOVA procedure. Results were considered significant at $p=0.05$.

\section{RESULTS}

\section{Morphological Development for Nauplii and Copepodites}

The major changes in morphology for each stage of nauplii and copepodite are listed in Tables 1 and 2.

TABLE 1

\begin{tabular}{|c|c|c|c|c|c|c|}
\hline Nauplii & $\begin{array}{c}\text { No. of Seta on } \\
\text { Last Segment } \\
\text { of Antenna } \\
\text { Prima }\end{array}$ & $\begin{array}{c}\text { Body } \\
\text { Length } \\
(\mathrm{mm})\end{array}$ & Copepodite & $\begin{array}{c}\text { No. } \\
\text { Prosome } \\
\text { Segments }\end{array}$ & $\begin{array}{c}\text { No. } \\
\text { Abdominal } \\
\text { Segments }\end{array}$ & $\begin{array}{l}\text { Length of } \\
\text { Thoracic } \\
\text { Segment (mm) }\end{array}$ \\
\hline $\mathrm{NI}$ & Last segment: 4 & $0.121-0.139$ & $\mathrm{Cl}$ & 3 & 2 & $0.325-0.425$ \\
\hline $\mathrm{NII}$ & Last segment: 4 & $0.161-0.17$ & Cll & 4 & 2 & $0.4674-0.5043$ \\
\hline NIII & $\begin{array}{l}\text { Abdominal: } 1 \\
\text { Last segment: } 4\end{array}$ & $0.190-0.22$ & CIII & 5 & 2 & $0.5576-0.63345$ \\
\hline NIV & $\begin{array}{c}\text { Dorsal: } 4 \\
\text { Abdominal: } 3 \\
\text { Last segment: } 4\end{array}$ & $0.225-0.25$ & CIV & 5 & 3 & $\begin{array}{c}0.79335-0.825(\mathrm{~F}) \\
0.6765-0.76875(\mathrm{M})\end{array}$ \\
\hline NV & $\begin{array}{c}\text { Dorsal: } 6 \\
\text { Abdominal: } 4 \\
\text { Last segment: } 4\end{array}$ & $0.271-0.30$ & CV & 5 & $\begin{array}{l}3(\mathrm{~F}) \\
4(\mathrm{M})\end{array}$ & $\begin{array}{c}0.933043-1.0332(\mathrm{~F}) \\
0.7995-0.861(\mathrm{M})\end{array}$ \\
\hline NVI & $\begin{array}{c}\text { Dorsal: } 8 \\
\text { Abdominal: } 4 \\
\text { Last segment: } 4\end{array}$ & $0.305-0.32$ & Adult & 4 & $\begin{array}{l}3(\mathrm{~F}) \\
4(\mathrm{M})\end{array}$ & $\begin{array}{c}1.0947-1.1931(\mathrm{~F}) \\
0.9471-1.04165(\mathrm{M})\end{array}$ \\
\hline
\end{tabular}

Main Characteristics of Each Stage of Nauplii and Copepodites of $P$. dubia

Note: $\mathrm{F}$, female; $\mathrm{M}$, male.

\section{Effect of Temperature on Development Time of Larval $P$. dubia}

When water temperature ranges from 15 to $35^{\circ} \mathrm{C}$, the development time required for each stage decreases as the temperature increases (Table 2, Figs. 1 and 2). At the nauplius stages, development time is minimal $(66 \mathrm{~h})$ at a water temperature of 30 to $35^{\circ} \mathrm{C}$. However, it takes $294 \mathrm{~h}$ to complete all of the nauplius stages at $15^{\circ} \mathrm{C}$. At the copepodite stages, it takes $114 \mathrm{~h}$ for copepodite stage I to develop into the adult stage at $35^{\circ} \mathrm{C}$, which is $520 \mathrm{~h}$ shorter than at $15^{\circ} \mathrm{C}$. The entire larval development time is $180 \mathrm{~h}$ at $35^{\circ} \mathrm{C}$ and $928 \mathrm{~h}$ at $15^{\circ} \mathrm{C}$. 
TABLE 2

Development Time (h) of Larvae of $P$. dubia under Different Temperatures

\begin{tabular}{lccccc}
\hline Larval Stage & $\mathbf{1 5}^{\circ} \mathbf{C}$ & $\mathbf{2 0}^{\circ} \mathbf{C}$ & $\mathbf{2 5}^{\circ} \mathbf{C}$ & $\mathbf{3 0}^{\circ} \mathbf{C}$ & $\mathbf{3 5}^{\circ} \mathbf{C}$ \\
\hline NII & 54 & 24 & 18 & 12 & 12 \\
NIII & 60 & 24 & 18 & 12 & 12 \\
NIV & 60 & 32 & 18 & 12 & 12 \\
NV & 60 & 32 & 18 & 12 & 12 \\
NVI & 60 & 32 & 24 & 18 & 18 \\
NII-NVI & 294 & 144 & 96 & 66 & 66 \\
CI & 80 & 30 & 24 & 18 & 18 \\
CII & 112 & 42 & 36 & 24 & 24 \\
CIII & 128 & 48 & 36 & 30 & 24 \\
CIV & 144 & 54 & 42 & 36 & 24 \\
CV & 170 & 66 & 42 & 36 & 24 \\
CI-CV & 634 & 240 & 180 & 144 & 114 \\
Sum (h) & 928 & 384 & 276 & 210 & 180 \\
\hline
\end{tabular}

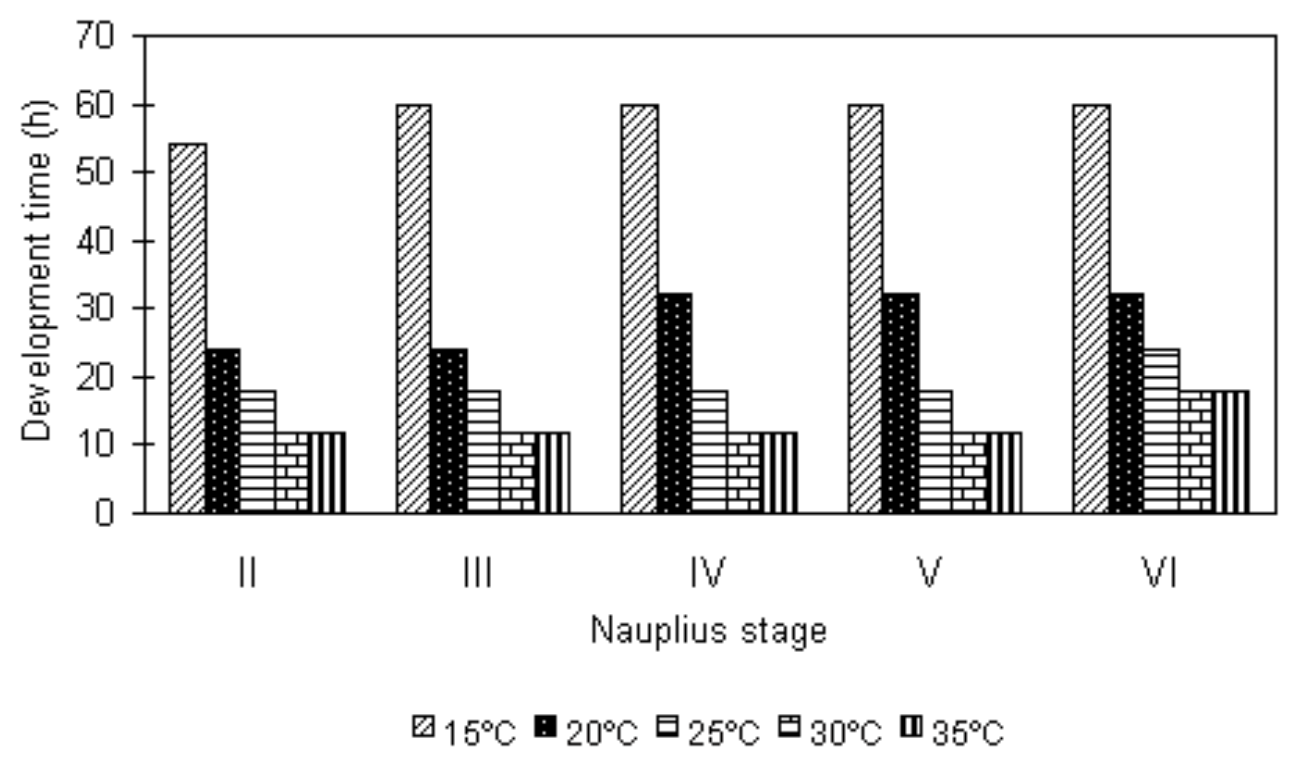

FIGURE 1. The development time of nauplii of $P$. dubia under different temperatures.

\section{Effect of Temperature on the Growth of P. dubia}

The effect of temperature on prosome length and weight for larvae of the same stage was minor (Tables 3 and 4). This is because prosome length and weight gains were insignificant at the naupliar stage. However, changes in weight at the copepodite stage were significant.

At a temperature range between 15 and $30^{\circ} \mathrm{C}$, the growth rate for larvae was consistently higher than that of larvae grown at a low temperature (Fig. 3 and 4). For example, the growth rate for CV at 35, 30, 25 , and $20^{\circ} \mathrm{C}$ was $6.35-, 3.72-, 3.05-$, and 2.81 -fold higher than the growth rate at $15^{\circ} \mathrm{C}$, indicating that at high temperatures, copepodite metabolism accelerated, leading to a higher growth rate. 


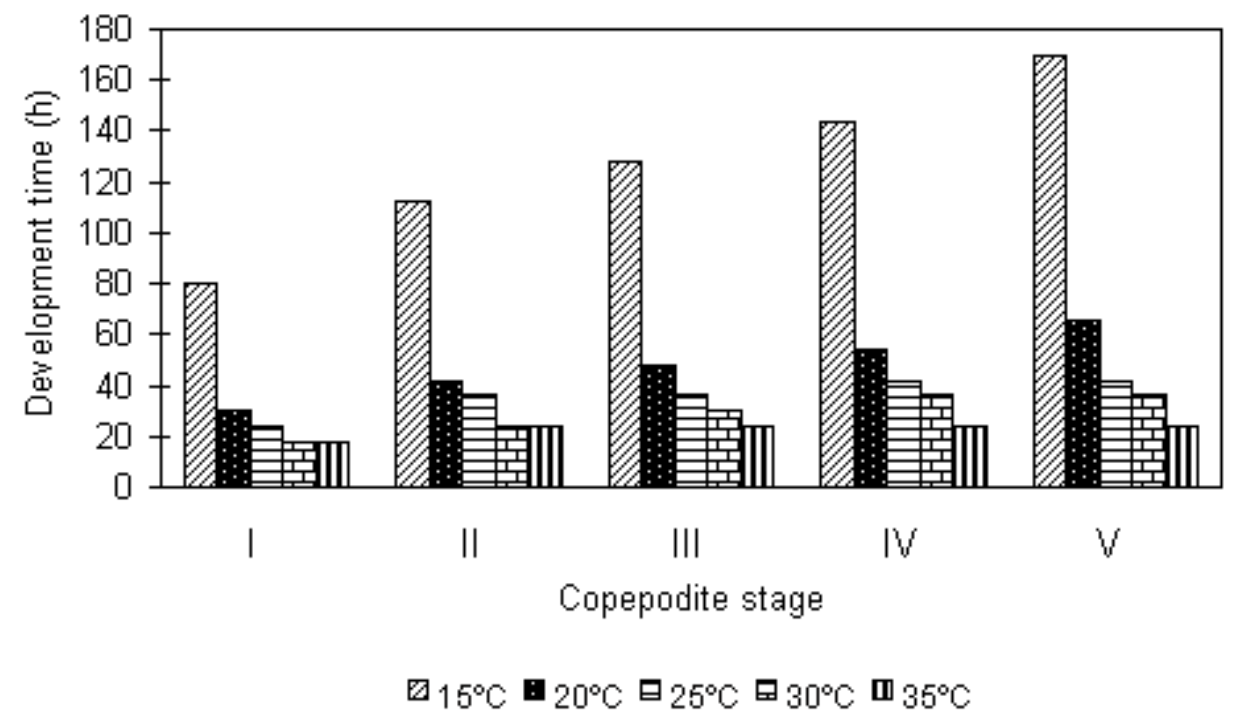

FIGURE 2. Development time of copepodites of $P$. dubia under different temperatures.

TABLE 3

Effects of Temperature on Development and Growth of the Naupliar Stages of $P$. dubia

\begin{tabular}{|c|c|c|c|c|c|c|}
\hline Instar & Parameter & $15^{\circ} \mathrm{C}$ & $20^{\circ} \mathrm{C}$ & $25^{\circ} \mathrm{C}$ & $30^{\circ} \mathrm{C}$ & $35^{\circ} \mathrm{C}$ \\
\hline \multirow[t]{4}{*}{$\mathrm{NII}$} & Prosome length $(\mu \mathrm{m})$ & 165 & 170 & 165 & 170 & 170 \\
\hline & Weight ( $\mu$ g C) & 0.0582 & 0.0618 & 0.0582 & 0.0618 & 0.0600 \\
\hline & Development time (days) & 2.25 & 1 & 0.75 & 0.5 & 0.5 \\
\hline & Growth rate ( $\mu \mathrm{g} \mathrm{C} / \mathrm{day})$ & 0.0201 & 0.0370 & 0.0517 & 0.0723 & 0.0759 \\
\hline \multirow[t]{4}{*}{ NIII } & Prosome length $(\mu \mathrm{m})$ & 220 & 215 & 213 & 214 & 216 \\
\hline & Weight ( $\mu \mathrm{g} \mathrm{C})$ & 0.1035 & 0.09883 & 0.097 & 0.0979 & 0.1 \\
\hline & Development time (days) & 2.5 & 1 & 0.75 & 0.5 & 0.5 \\
\hline & Growth rate ( $\mu \mathrm{g} \mathrm{C} /$ day) & 0.01206 & 0.0295 & 0.0390 & 0.0464 & 0.0346 \\
\hline \multirow[t]{4}{*}{ NIV } & Prosome length $(\mu \mathrm{m})$ & 250 & 245 & 243 & 238 & 234 \\
\hline & Weight ( $\mu \mathrm{g} \mathrm{C})$ & 0.1336 & 0.1283 & 0.1262 & 0.1211 & 0.12 \\
\hline & Development time (days) & 2.5 & 1.3333 & 0.75 & 0.5 & 0.5 \\
\hline & Growth rate ( $\mu \mathrm{g} \mathrm{C} /$ day) & 0.0160 & 0.0206 & 0.0536 & 0.1028 & 0.1035 \\
\hline \multirow[t]{4}{*}{ NV } & Prosome length $(\mu \mathrm{m})$ & 285 & 270 & 279 & 284 & 281 \\
\hline & Weight ( $\mu \mathrm{g} \mathrm{C})$ & 0.1737 & 0.1559 & 0.1664 & 0.1724 & 0.17 \\
\hline & Development time (days) & 2.5 & 1.3333 & 0.75 & 0.5 & 0.5 \\
\hline & Growth rate ( $\mu \mathrm{g} \mathrm{C} /$ day) & 0.0209 & 0.0483 & 0.0774 & 0.0957 & 0.0975 \\
\hline \multirow[t]{4}{*}{ NVI } & Prosome length $(\mu \mathrm{m})$ & 325 & 321 & 324 & 321 & 319 \\
\hline & Weight ( $\mu \mathrm{g} \mathrm{C})$ & 0.2258 & 0.2203 & 0.2244 & 0.2203 & 0.22 \\
\hline & Development time (days) & 2.5 & 1.3333 & 1 & 0.75 & 0.75 \\
\hline & Growth rate ( $\mu \mathrm{g} \mathrm{C} /$ day) & 0.0605 & 0.0603 & 0.0527 & 0.1179 & 0.1108 \\
\hline
\end{tabular}


TABLE 4

Effects of Temperature on Development and Growth of Copepodite Stage of $P$. dubia

\begin{tabular}{|c|c|c|c|c|c|c|}
\hline Instar & Parameter & $15^{\circ} \mathrm{C}$ & $20^{\circ} \mathrm{C}$ & $25^{\circ} \mathrm{C}$ & $30^{\circ} \mathrm{C}$ & $35^{\circ} \mathrm{C}$ \\
\hline \multirow[t]{4}{*}{$\mathrm{Cl}$} & Prosome length $(\mu \mathrm{m})$ & 420 & 375 & 360 & 380 & 375 \\
\hline & Weight ( $\mu \mathrm{g} C)$ & 0.2195 & 0.1596 & 0.1423 & 0.1657 & 0.16 \\
\hline & Development time (days) & 3.3333 & 1.25 & 1 & 0.75 & 0.75 \\
\hline & Growth rate ( $\mu \mathrm{g} \mathrm{C} /$ day $)$ & 0.04424 & 0.1274 & 0.2000 & 0.2355 & 0.1824 \\
\hline \multirow[t]{4}{*}{ Cll } & Prosome length $(\mu \mathrm{m})$ & 504.3 & 479.7 & 492 & 492 & 467 \\
\hline & Weight ( $\mu \mathrm{g} \mathrm{C})$ & 0.3669 & 0.3188 & 0.3423 & 0.3423 & 0.3 \\
\hline & Development time (days) & 4.6667 & 1.75 & 1.5 & 1 & 1 \\
\hline & Growth rate ( $\mu \mathrm{g} \mathrm{C} /$ day) & 0.0587 & 0.1997 & 0.1960 & 0.1647 & 0.1902 \\
\hline \multirow[t]{4}{*}{ CIII } & Prosome length $(\mu \mathrm{m})$ & 615 & 614.225 & 613.45 & 565.8 & 558 \\
\hline & Weight ( $\mu \mathrm{g} C)$ & 0.6408 & 0.6682 & 0.6963 & 0.5070 & 0.49 \\
\hline & Development time (days) & 5.3333 & 2 & 1.5 & 1.25 & 1 \\
\hline & Growth rate ( $\mu \mathrm{g} \mathrm{C} /$ day) & 0.0828 & 0.2133 & 0.3185 & 0.3353 & 0.3510 \\
\hline \multirow[t]{4}{*}{ CIV } & Prosome length $(\mu \mathrm{m})$ & 741.075 & 744.15 & 768.75 & 701.1 & 677 \\
\hline & Weight ( $\mu \mathrm{g} \mathrm{C)}$ & 1.0822 & 1.0949 & 1.114 & 0.9261 & 0.84 \\
\hline & Development time (days) & 6 & 2.25 & 1.75 & 1.5 & 1 \\
\hline & Growth rate ( $\mu \mathrm{g} \mathrm{C} /$ day) & 0.0837 & 0.2465 & 0.2687 & 0.4387 & 0.6829 \\
\hline \multirow[t]{4}{*}{ CV } & Prosome length $(\mu \mathrm{m})$ & 848.7 & 861 & 848.7 & 848.7 & 836 \\
\hline & Weight ( $\mu \mathrm{g} \mathrm{C})$ & 1.5842 & 1.6495 & 1.5842 & 1.5842 & 1.52 \\
\hline & Development time (days) & 7.0833 & 2.75 & 1.75 & 1.5 & 1 \\
\hline & Growth rate ( $\mu \mathrm{g} \mathrm{C} /$ day) & 0.1114 & 0.4246 & 0.4517 & 0.5261 & 0.8189 \\
\hline
\end{tabular}

Note: Body lengths for CIV-CV were based on the body length of males.

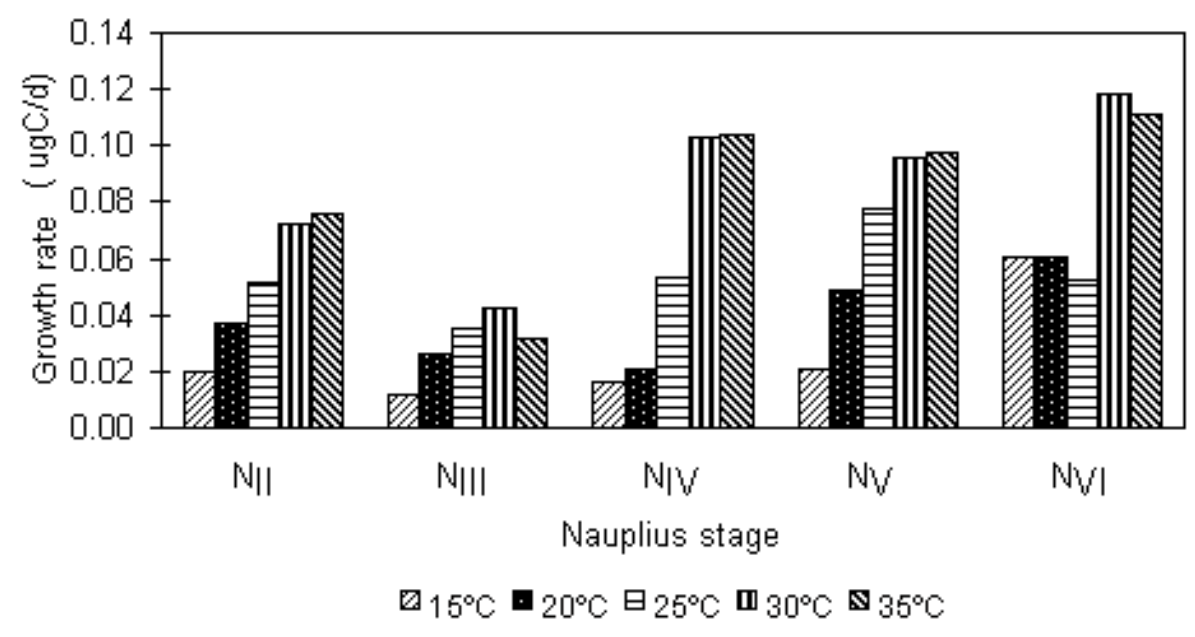

FIGURE 3. Effect of temperature on growth rate of $P$. dubia nauplii. 


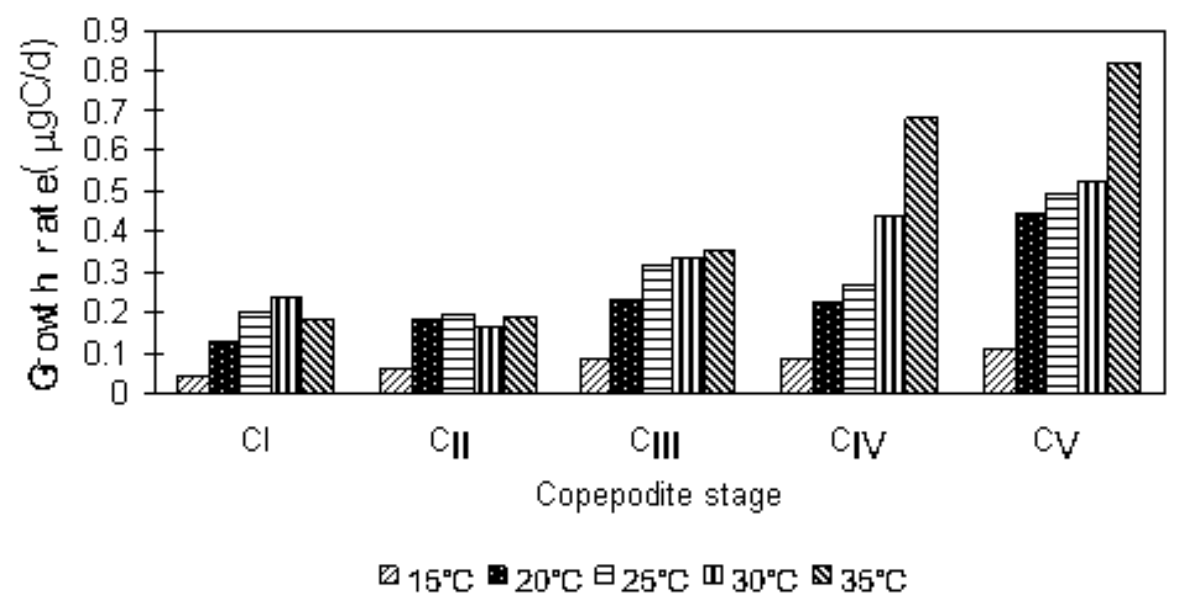

FIGURE 4. Effect of temperature on growth rate of $P$. dubia copepodites.

\section{Effects of Temperature on Larval Survival Rate}

The effects of temperature on the survival rate of juvenile $P$. dubia were significant (Fig. 5 and Table 5). Within the suitable range, the larval survival rate at high temperatures was always higher than that at low temperatures. The survival rates for both nauplii and copepodites were highest at a temperature of $30^{\circ} \mathrm{C}$ and were 80 and $67.5 \%$, respectively, and decreased when the temperature exceeded $30^{\circ} \mathrm{C}$. The survival rate for nauplii at 30 and $35^{\circ} \mathrm{C}$ was significantly higher than at 15 and $20^{\circ} \mathrm{C}(p<0.05)$; the survival rate for copepodites at 20 and $35^{\circ} \mathrm{C}$ was significantly higher than at $15^{\circ} \mathrm{C}(p<0.05)$, with the survival rate at $30^{\circ} \mathrm{C}$ 1.45 -fold of that at $15^{\circ} \mathrm{C}$. The most suitable growth temperature for $P$. dubia ranged from 20 to $35^{\circ} \mathrm{C}$.

\section{Effects of Temperature on Reproduction}

The life span and hatching time for $P$. dubia decreased as the temperature increased, and were lowest at $35^{\circ} \mathrm{C}$ (41 and 1.02 days, respectively), which were about 64 and $60 \%$ less than those at $20^{\circ} \mathrm{C}$ (Table 6). When the temperature ranged from 20 to $30^{\circ} \mathrm{C}$, the total larval production and reproduction time increased as the temperature increased, but decreased when the temperature exceeded $30^{\circ} \mathrm{C}$. The highest average larval production and reproduction frequency appeared at $30^{\circ} \mathrm{C}$, accounting for 480 individuals/female and 30 times of reproduction, followed at $25^{\circ} \mathrm{C}$. Therefore, the optimal reproduction temperature ranged from 25 to $30^{\circ} \mathrm{C}$.

\section{Effects of Temperature on Reproduction and Growth Parameters}

The intrinsic growth rate, finite growth rate, and net growth rate were $0.195,1.215,264$, respectively, at $30^{\circ} \mathrm{C}$ (Tables 7 and 8$)$, followed by $25^{\circ} \mathrm{C}(0.168,1.183$, and 163$)$ and $35^{\circ} \mathrm{C}(0.079,1.082$, and 3.3$)$. At temperatures between 25 and $30^{\circ} \mathrm{C}$, the population doubling time was 3.556 to 4.128 days, which were less than those at 20 and $35^{\circ} \mathrm{C}$. However, the generation time decreased as the temperature increased, with the shortest at $25^{\circ} \mathrm{C}$ (15.36 days) and longest at $20^{\circ} \mathrm{C}$ (58.5 days). The optimal temperatures for population growth ranged from 25 to $30^{\circ} \mathrm{C}$. 


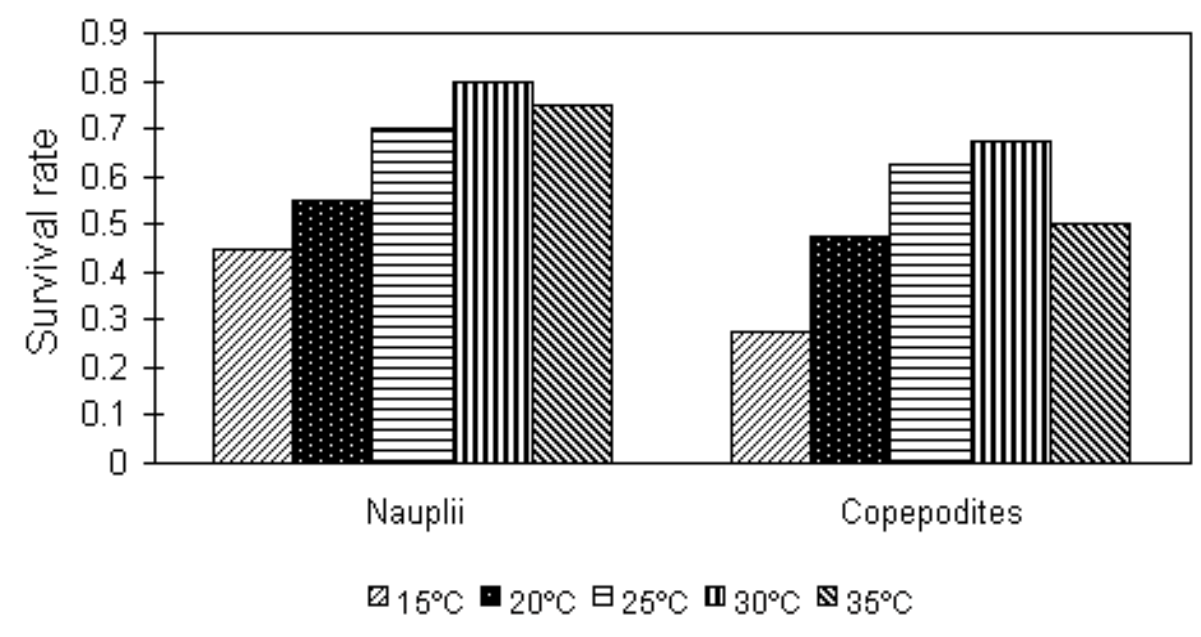

FIGURE 5. Effect of temperature on survival rate of $P$. dubia.

TABLE 5

Variance Analysis of Survival Rate of $\boldsymbol{P}$. dubia under Different Temperatures

\begin{tabular}{llccccc}
\hline Variance & & SS & $\boldsymbol{d f}$ & $\mathbf{M s}$ & $\mathbf{F}$ & $\mathbf{F}_{0.05}$ \\
\hline Nauplii & Treatment & 0.17 & 4 & 0.0425 & $6.0714^{*}$ & 3.06 \\
& Error & 0.035 & 5 & 0.007 & & \\
& Total variance & 0.205 & 9 & & & \\
Copepodites & Treatment & 0.388 & 4 & 0.097 & $4.4091^{*}$ & 3.06 \\
& Error & 0.33 & 5 & 0.022 & & \\
& Total variance & 0.718 & 9 & & & \\
\hline
\end{tabular}

* Significant at $p<0.05$.

TABLE 6

Growth and Production Parameters of $\boldsymbol{P}$. dubia under Different Temperatures

\begin{tabular}{lcccc}
\hline Growth/Reproduction Indices & \multicolumn{4}{c}{ Temperature $\left({ }^{\circ} \mathbf{C}\right)$} \\
\cline { 2 - 5 } & $\mathbf{2 0}$ & $\mathbf{2 5}$ & $\mathbf{3 0}$ & $\mathbf{3 5}$ \\
\hline Development stage before hatching (days) & 16 & 11.5 & 8.75 & 7.5 \\
Reproduction stage (day) & 100.2 & 54 & 46.5 & 33.8 \\
Range of longevity (days) & $84-119$ & $41-79$ & $37-64$ & $39-45$ \\
Average longevity (days) & 116.2 & 65.5 & 55.3 & 41.3 \\
Range of total larval production (larvae/female) & $72-467$ & $199-447.5$ & $255-583$ & $1-20$ \\
Average larval production (larvae/female) & 195.13 & 316.75 & 480.25 & 3.88 \\
Reproduction frequency & 20 & 26 & 30 & 0.5 \\
Average clutch size & 9.58 & 12.18 & 16.00 & 7.75 \\
Hatching time (days) & 2.60 & 2.05 & 1.2 & 1.02 \\
\hline
\end{tabular}


TABLE 7

Fecundity Life Table for $\boldsymbol{P}$. dubia at Different Temperatures

\begin{tabular}{|c|c|c|c|c|c|c|c|c|c|c|c|c|c|c|c|c|}
\hline \multirow{2}{*}{$\begin{array}{l}\text { Age, } x \\
\text { (days) }\end{array}$} & \multicolumn{4}{|c|}{ Survival Rate $\mathrm{I}_{\mathrm{x}}\left({ }^{\circ} \mathrm{C}\right)$} & \multicolumn{4}{|c|}{ Birth Rate $\mathrm{m}_{\mathrm{x}}\left({ }^{\circ} \mathrm{C}\right)$} & \multicolumn{4}{|c|}{$\mathrm{I}_{\mathrm{x}} \mathrm{m}_{\mathrm{x}}\left({ }^{\circ} \mathrm{C}\right)$} & \multicolumn{4}{|c|}{$\left.x\right|_{x} m_{x}\left({ }^{\circ} \mathrm{C}\right)$} \\
\hline & 20 & 25 & 30 & 35 & 20 & 25 & 30 & 35 & 20 & 25 & 30 & 35 & 20 & 25 & 30 & 35 \\
\hline 1 & 1 & 1 & 1 & 1 & 0 & 0 & 0 & 0 & & & & & & & & \\
\hline 3 & & 0.75 & 0.8 & 0.75 & 0 & 0 & 0 & 0 & & & & & & & & \\
\hline 6 & 0.6 & & 0.68 & 0.45 & & 0 & 0 & 0 & & 0 & & & & 0 & & \\
\hline 11 & 0.6 & 0.63 & 0.68 & 0.45 & 0 & 0 & 9.71 & 3 & 0 & 0 & 6.56 & 1.35 & 0 & 0 & 72.1 & 14.9 \\
\hline 12 & 0.6 & 0.63 & 0.68 & 0.45 & 0 & 6.67 & 4.43 & 0 & 0 & 4.17 & 2.99 & 0 & 0 & 50 & 35.9 & 0 \\
\hline 13 & 0.6 & 0.63 & 0.68 & 0.45 & 0 & 7.67 & 8.71 & 2 & 0 & 4.79 & 5.88 & 0.9 & 0 & 62.3 & 76.5 & 11.7 \\
\hline 14 & 0.6 & 0.63 & 0.68 & 0.45 & 0 & 5.75 & 0 & 0 & 0 & 3.59 & 0 & 0 & 0 & 50.3 & 0 & 0 \\
\hline 15 & 0.6 & 0.63 & 0.68 & 0.45 & 0 & 9.83 & 12.3 & 0 & 0 & 6.15 & 8.29 & 0 & 0 & 92.2 & 124 & 0 \\
\hline 17 & 0.48 & 0.63 & 0.68 & 0.45 & 0 & 16.3 & 33.7 & 0 & 0 & 10.2 & 22.8 & 0 & 0 & 174 & 387 & 0 \\
\hline 18 & 0.48 & 0.63 & 0.68 & 0.45 & 0 & 0 & 17.6 & 1 & 0 & 0 & 11.9 & 0.45 & 0 & 0 & 214 & 8.1 \\
\hline 19 & 0.48 & 0.63 & 0.68 & 0.45 & 0 & 12.2 & 14.3 & 0 & 0 & 7.6 & 9.68 & 0 & 0 & 145 & 184 & 0 \\
\hline 21 & 0.48 & 0.63 & 0.68 & 0.45 & 0 & 12.5 & 32.5 & 0 & 0 & 7.81 & 21.9 & 0 & 0 & 164 & 461 & 0 \\
\hline 22 & 0.48 & 0.63 & 0.68 & 0.45 & 3.38 & 3.33 & 18.8 & 0 & 1.6 & 2.08 & 12.7 & 0 & 35.3 & 45.8 & 280 & 0 \\
\hline 24 & 0.46 & 0.59 & 0.63 & 0.45 & 5.88 & 14.8 & 18.8 & 0 & 2.67 & 8.73 & 11.8 & 0 & 64.2 & 209 & 284 & 0 \\
\hline 26 & 0.46 & 0.59 & 0.63 & 0.45 & 8.88 & 16.5 & 37.8 & 0 & 4.04 & 9.71 & 23.8 & 0 & 105 & 252 & 619 & 0 \\
\hline 27 & 0.46 & 0.59 & 0.63 & 0.32 & 9.75 & 3.17 & 17.8 & 2 & 4.44 & 1.86 & 11.2 & 0.64 & 120 & 50.3 & 302 & 16.6 \\
\hline 29 & 0.46 & 0.59 & 0.57 & 0.28 & 0 & 12.2 & 15.8 & 0 & 0 & 7.16 & 9.02 & 0 & 0 & 208 & 262 & 0 \\
\hline 31 & 0.46 & 0.59 & 0.52 & 0.28 & 14.1 & 17.7 & 36.3 & & 6.43 & 10.4 & 18.7 & & 199 & 322 & 579 & \\
\hline 33 & 0.46 & 0.59 & 0.52 & 0.24 & 11.6 & 10.3 & 18.5 & 0 & 5.29 & 6.08 & 9.54 & 0 & 175 & 201 & 315 & 0 \\
\hline 35 & 0.46 & 0.55 & 0.52 & 0.2 & 6.38 & 15 & 19 & 0 & 2.9 & 8.27 & 9.8 & 0 & 102 & 290 & 343 & 0 \\
\hline 37 & 0.46 & 0.55 & 0.46 & 0.12 & 9 & 17.3 & 25 & 0 & 4.1 & 9.56 & 11.5 & 0 & 152 & 354 & 424 & 0 \\
\hline 39 & 0.46 & 0.48 & 0.46 & 0.1 & 6.38 & 13.5 & 19.7 & 0 & 2.9 & 6.45 & 9.01 & 0 & 113 & 252 & 351 & 0 \\
\hline 41 & 0.46 & 0.48 & 0.46 & 0.05 & 13.5 & 15.6 & 12.7 & 0 & 6.15 & 7.46 & 5.8 & 0 & 252 & 306 & 238 & 0 \\
\hline 43 & 0.46 & 0.44 & 0.39 & 0.05 & 8.5 & 13.6 & 20.7 & 0 & 3.87 & 6 & 8.12 & 0 & 166 & 258 & 349 & 0 \\
\hline 45 & 0.46 & 0.44 & 0.39 & 0.03 & 5 & 4.4 & 22 & 0 & 2.28 & 1.94 & 8.64 & 0 & 102 & 87.4 & 389 & 0 \\
\hline 47 & 0.46 & 0.37 & 0.33 & 0 & 10.6 & 15 & 17 & 0 & 4.84 & 5.52 & 5.56 & 0 & 227 & 259 & 262 & 0 \\
\hline 49 & 0.46 & 0.29 & 0.33 & & 7.63 & 16 & 13.3 & & 3.47 & 4.71 & 4.36 & & 170 & 231 & 214 & \\
\hline 50 & 0.46 & 0.29 & 0.33 & & 2.63 & 8.4 & 5.33 & & 1.2 & 2.47 & 1.75 & & 59.8 & 124 & 87.3 & \\
\hline 52 & 0.46 & 0.29 & 0.33 & & 0 & 13 & 9.67 & & & 3.82 & 3.16 & & & 199 & 165 & \\
\hline 53 & 0.46 & 0.24 & 0.26 & & 10.3 & 7.8 & 6 & & 4.67 & 1.86 & 1.57 & & 247 & 98.8 & 83.3 & \\
\hline 56 & 0.46 & 0.24 & 0.26 & & 6.25 & 15 & 14.3 & & 2.85 & 3.59 & 3.75 & & 159 & 201 & 210 & \\
\hline 59 & 0.46 & 0.24 & 0.2 & & 13 & 25.5 & 18 & & 5.92 & 6.09 & 3.53 & & 349 & 360 & 209 & \\
\hline 61 & 0.46 & 0.24 & 0.13 & & 4 & 5.33 & 3 & & 1.82 & 1.28 & 0.39 & & 111 & 77.8 & 24 & \\
\hline 64 & 0.46 & 0.24 & 0 & & 7.67 & 7.33 & 0 & & 3.49 & 1.75 & 0 & & 223 & 112 & 0 & \\
\hline 66 & 0.44 & 0.21 & & & 6.5 & 7.33 & & & 2.83 & 1.53 & & & 187 & 101 & & \\
\hline 68 & 0.42 & 0.12 & & & 3.88 & 3.67 & & & 1.61 & 0.44 & & & 110 & 29.8 & & \\
\hline 70 & 0.42 & 0.09 & & & 5.13 & 3 & & & 2.15 & 0.27 & & & 151 & 18.8 & & \\
\hline 71 & 0.4 & 0.03 & & & 4 & 0.33 & & & 1.6 & 0.01 & & & 114 & 0.71 & & \\
\hline 74 & 0.4 & 0.03 & & & 8.75 & 2.5 & & & 3.5 & 0.08 & & & 259 & 5.53 & & \\
\hline 77 & 0.4 & 0.03 & & & 7 & 0 & & & 2.8 & 0 & & & 216 & 0 & & \\
\hline 79 & 0.4 & 0 & & & 2.63 & 0 & & & 1.05 & 0 & & & 83 & 0 & & \\
\hline 84 & 0.36 & & & & 19.9 & & & & 7.15 & & & & 601 & & & \\
\hline 89 & 0.34 & & & & 10.9 & & & & 3.69 & & & & 329 & & & \\
\hline 91 & 0.34 & & & & 10.5 & & & & 3.57 & & & & 325 & & & \\
\hline 94 & 0.32 & & & & 6.83 & & & & 2.19 & & & & 206 & & & \\
\hline 98 & 0.32 & & & & 7.33 & & & & 2.35 & & & & 230 & & & \\
\hline 103 & 0.32 & & & & 10.7 & & & & 3.41 & & & & 352 & & & \\
\hline 104 & 0.32 & & & & 2.5 & & & & 0.8 & & & & 83.2 & & & \\
\hline 105 & 0.28 & & & & 0 & & & & 0 & & & & 0 & & & \\
\hline 106 & 0.28 & & & & 1 & & & & 0.28 & & & & 29.7 & & & \\
\hline
\end{tabular}


TABLE 7 (continued)

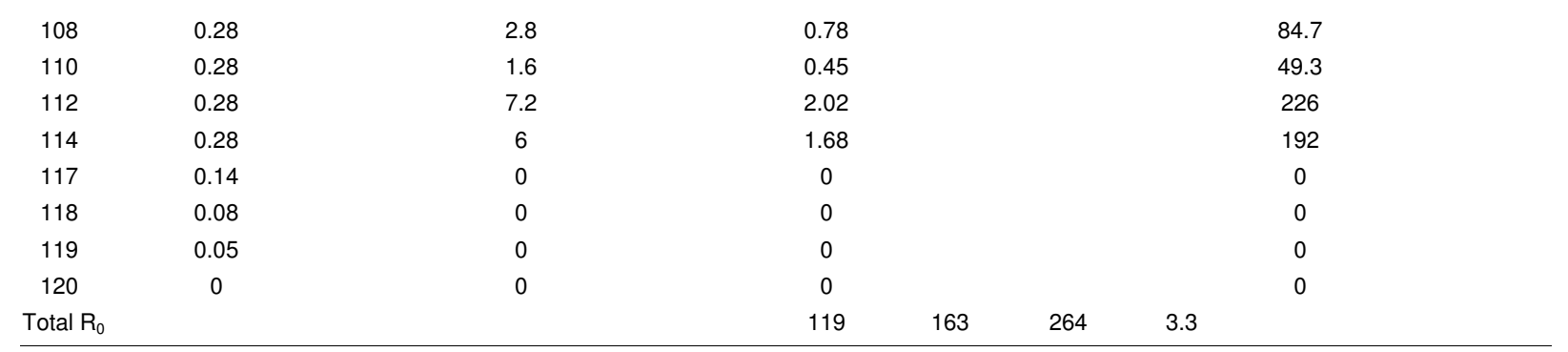

TABLE 8

Life Table Parameters of $\boldsymbol{P}$. dubia at Different Temperatures

\begin{tabular}{lcccc}
\hline \multirow{2}{*}{ Growth Parameters } & \multicolumn{4}{c}{ Temperature (ㅇ) } \\
\cline { 2 - 5 } & $\mathbf{2 0}$ & $\mathbf{2 5}$ & $\mathbf{3 0}$ & $\mathbf{3 5}$ \\
\hline Net reproduction rate $\left(\mathrm{R}_{0}\right)$ & 119 & 163 & 264 & 3.3 \\
Population growth cycle $(\mathrm{T})$ & 58.5 & 30.4 & 28.6 & 15.36 \\
Intrinsic growth rate $\left(\mathrm{r}_{\mathrm{m}}\right)$ & 0.082 & 0.168 & 0.195 & 0.079 \\
Population doubling time $(\mathrm{t})$ & 8.49 & 4.13 & 3.56 & 8.833 \\
Finite growth rate $(\lambda)$ & 1.085 & 1.183 & 1.215 & 1.082 \\
\hline
\end{tabular}

\section{Effects of Temperature on Generation Survival Curve}

The generation survival curve of $P$. dubia was steepest and shortest at $35^{\circ} \mathrm{C}$ (Fig. 6), but was similar between 25 and $30^{\circ} \mathrm{C}$, and longest at $20^{\circ} \mathrm{C}$, which corresponded to their life cycle.

\section{DISCUSSION}

\section{Effects of Temperature on Juvenile Development and Survival}

Water temperature is an important factor that affects the survival, development, and growth of aquatic invertebrates[5,13]. Temperature affects metabolism level and oxygen consumption that, in turn, affect an animal's ability to resist environmental changes. High temperatures can accelerate metabolism and oxygen consumption, leading to excessive use of protein and a low survival rate[14,15,16]. This is in agreement with our findings that indicate significant effects of temperature on development time, survival rate, and growth rates of $P$. dubia. As the temperature increases, the development time required for each larval stage decreases and growth rates increase. The survival rate increases as the temperature increases within the range from 15 to $30^{\circ} \mathrm{C}$. However, when the temperature exceeds $35^{\circ} \mathrm{C}$, the larval survival rate begins to decline and larval development slows down as the temperature drops. During copepod development, there are three critical stages, i.e., fertilized egg to nauplii, NIII to NIV, and NVI to CI, which are extremely sensitive to environmental changes as evidenced by high mortality. These periods will be prolonged if development slows down, leading to high mortality. At a temperature of $15^{\circ} \mathrm{C}$, the entire larval development time is 33 days. This explains the high mortality under low temperatures, which delay larval development significantly. Therefore, temperature is a critical factor that affects the copepod life cycle. 


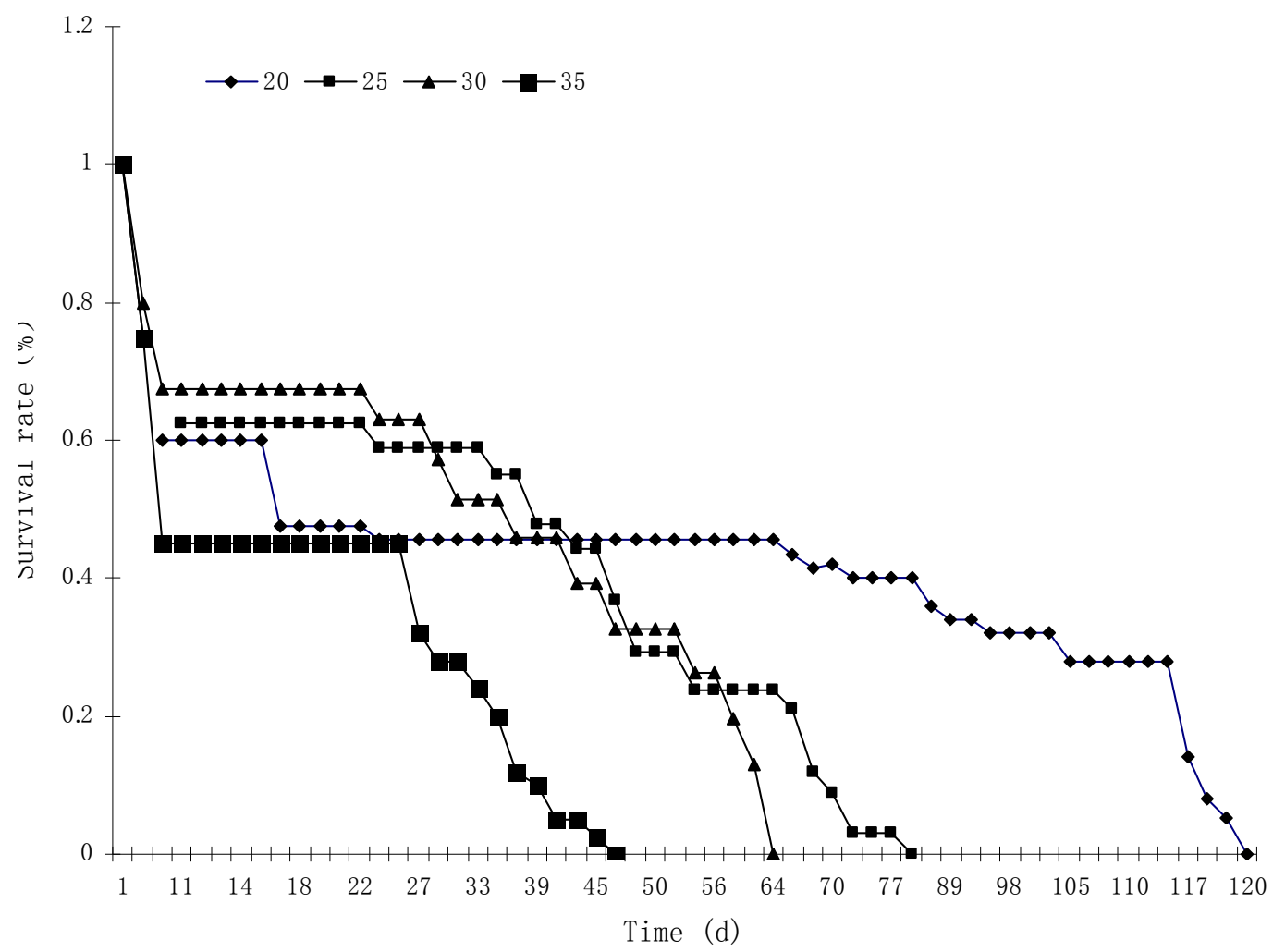

FIGURE 6. Survival curves of $P$. dubia at different temperatures.

Within the experimental range of temperature, there was no difference in body weight between nauplii and copepodites. Previous studies found that adult body weight can attain its maximum under low temperatures[17] and large individuals require a longer period of development[16]. This finding is contradictory to our results, which revealed no significant difference in body length for the same larval stage under different temperatures. We also did not observe an increase in body length with a decrease in water temperature in our experiment.

\section{Effects of Temperature on Reproduction}

Chen[18] reported an increase in mating frequency under high temperatures. However, negative effects on the reproduction of copepods have also been reported. For example, Li and Chen[19] indicated in their experiment that the egg production increased as the temperature increased and reached its peak at $24^{\circ} \mathrm{C}$, and then decreased with further increase in temperature. McKinnon[20] also found that the body length of a subtropical copepod, Acrocalanus gibber, increased with water temperature. These experiments indicate that an optimal spectrum of temperature is species specific[14]. This is in agreement with our results that indicate the importance of temperature to the longevity and reproduction of $P$. dubia. As temperature increases, the longevity and hatching time for $P$. dubia decrease. Total egg production and mating rate increase as temperature increases, but decreases when the temperature exceeds $30^{\circ} \mathrm{C}$. Hatching rate and reproduction frequency are at the lowest at $35^{\circ} \mathrm{C}$. Therefore, within a suitable temperature range, elevated temperature may promote copepod reproduction, increase egg production, and shorten reproduction cycle and hatching time. 
Chen[18] found that at high temperatures, mating individuals increased and mating time decreased. Our experiment indicates that the optimal temperature ranges from 25 to $30^{\circ} \mathrm{C}$ for population growth and reproduction by $P$. dubia. Intrinsic, finite, and net growth rates are the highest at $30^{\circ} \mathrm{C}$. Meanwhile, the individual development and reproduction (clutch size and hatching rate) are also high. At $30^{\circ} \mathrm{C}$, it takes $210 \mathrm{~h}$ or 8.75 days for NII to reach the adult stage, with a survival rate of $80 \%$, an average longevity of 55.25 days, an average total hatching number of 480.21 /female, and a reproduction frequency of 30 with an average hatching of 16 larvae. Under the optimal temperature of $30^{\circ} \mathrm{C}$, the net production rate $\left(\mathrm{R}_{0}\right)$ is 264 , generation time $(T)$ is 28.6 days, intrinsic growth rate $\left(r_{m}\right)$ is 0.195 , finite growth rate $(\lambda)$ is 1.215 , and population doubling time $(\mathrm{t})$ is 3.55 . These data suggest a very high reproduction capacity of $P$. dubia under the optimal temperature and food conditions.

Banse[21] found that intrinsic growth rate varied among different sizes of invertebrates at the same temperature. Ji et al.[22] found intrinsic growth rate of acarus (Oligonychus biharensis Hirstwas) at $35^{\circ} \mathrm{C}$ also higher than at $25^{\circ} \mathrm{C}$, but net reproduction rate and average egg production at $35^{\circ} \mathrm{C}$ were lower than at $25^{\circ} \mathrm{C}$, which led to a conclusion that $25^{\circ} \mathrm{C}$ is a more suitable temperature for $O$. biharensis. Our results show little difference in intrinsic growth rate at $35^{\circ} \mathrm{C}(0.79)$ and $20^{\circ} \mathrm{C}(0.082)$. However, this does not imply that there was no difference in population growth between these temperatures. At $35^{\circ} \mathrm{C}$, the reproduction rate of the next generation is 3.875 (Table 8), while the net reproduction rate at $20^{\circ} \mathrm{C}$ is 119 ; the average hatching rate is 3.875 larvae/female at $35^{\circ} \mathrm{C}$, which is significantly lower than the 195.125 larvae/female at $20^{\circ} \mathrm{C}$. It is concluded that population reproduction and growth are significantly better at $20^{\circ} \mathrm{C}$ than at $35^{\circ} \mathrm{C}$.

\section{ACKNOWLEDGMENTS}

This study was funded by the Ministry of Science and Technology of China (2006GB2E000224) and Guangdong Provincial Science and Technology Projects (2006A20305003).

\section{REFERENCES}

1. Uye, S. (1988) Temperature-dependent development and growth of Calanus sinicus (Copepoda: Calanoida) in the laboratory. Hydrobiologia 167, 285-293.

2. Haq, S.M. (1972) Breeding of Euterpina acutifrons, a harpacticoid copepod, with special reference to dimorphic males. Mar. Biol. 15, 221-235.

3. Hart, R.C. and McLaren, I.A. (1978) Temperature acclimation and other influences on embryonic duration in the copepod Pseudocalanus sp. Mar. Biol. 45, 23-30.

4. Heip, C. (1974) A comparison between models describing the influence of temperature on the development rate of copepods. Biol. Jb. Dodonaea 42, 121-125.

5. Hirst, A.G. and Bunker, A.J. (2003) Growth of marine planktonic copepods: global rates and patterns in relation to chlorophyll a, temperature, and body weight. Limnol. Oceanogr. 48, 1988-2010.

6. Peterson, W.T., Tiselius, P., and Kirboe, T. (1991) Copepod egg production, moulting and growth rates, and secondary production, in the Skagerrak in August 1988. J. Plankton Res. 13, 131-154.

7. Vidal, J. (1980) Physioecology of zooplankton. II. Effects of phytoplankton concentration, temperature, and body size on the development and molting rates of Calanus pacificus and Pseudocalanus sp. Mar. Biol. 56, 135-146.

8. Walter, T.C., Ohtsuka, S., and Castillo, L.V. (2006) A new species of Pseudodiaptomus (Crustacea: Copepoda: Calanoida) from the Philippines, with a key to pseudodiaptomids from the Philippines and comments on the status of the genus Schmackeria. Proc. Biol. Soc. Washington 119, 202-221.

9. Li, C., Luo, X., Huang, X., and Gu, B. (2008) Effects of temperature, salinity, pH, and light on filtering and grazing rates of a calanoid copepod (Schmackeria dubia). TheScientificWorldJOURNAL 8, 1219-1227.

10. Hong, H., Hu, Q., and Wu, Y. (1981) Marine Plankton Biology. Agriculture Press, Beijing. [Chinese]

11. Shen, G. (2002) Marine Ecology. Science Press, Beijing. [Chinese]

12. Tang, B., Zhang, F., Hu, Z., Xiong, J., and Geng, X. (2005) The comparison of development and life table of population between Tetranychus cinnabarinus (Boisduva1) and T. urticae (Koch). J. Mount. Agr. Biol. 24, 42-47. [Chinese] 
13. Campbell, R.G., Wagner, M.M., Teegarden, G.J., Boudreau, C.A., and Durbin, E.G. (2001) Growth and development rates of the copepod Calanus finmarchicus reared in the laboratory. Mar. Ecol. Prog. Ser. 221, 161-183.

14. Huntley, M.E. and Lopez, M.D.G. (1992) Temperature-dependent production of marine copepods: a global synthesis. Am. Nat. 140, 201-242.

15. Klein Breteler, W.C.M., Gonzalez, S.R., and Schogt, N. (1995) Development of Pseudocalanus elongates (Copepoda,Calanoida) cultured at different temperature and food conditions. Mar. Ecol. Prog. Ser. 199, 99-110.

16. Hirst, A.G. and Kioerboe, T. (2002) Mortality of marine planktonic copepods: global rates and patterns. Mar. Ecol. Prog. Ser. 230, 195-209.

17. Zheng, Z. (1993) Zheng Zhong Scientific Article Collection. Ocean Press, Beijing. [Chinese]

18. Chen, S.J. (1988) An indoor culture study of Euierpe acutifrons from Xiamen Bay. J. Xiamen Fish. Coll. 12, 339345. [Chinese]

19. Li, S. and Chen, F. (1989) Studies on the feature of eggs and its hatching rates of some plankton copepods in Xiamen waters. J. Xiamen Univ. (Nat. Sci.) 28, 538-543. [Chinese]

20. McKinnon, A.D. (1996) Growth and development in the subtropical copepod. Limnol. Oceanogr. 41, $1438-1447$.

21. Banse, K. (1982) Mass-scaled rates of respiration and intrinsic growth in very small invertebrates. Mar. Ecol. Prog. Ser. 9, 281-297.

22. Ji, J., Zhang, Y., Chen, X., and Lin, J. (2005) Laboratory population life table of Oligonychus biharensis (Hirst) (Acari:Tetranychidae) at different temperatures. Acta Arachnol. Sin. 14, 37-41. [Chinese]

\section{This article should be cited as follows:}

Li, C., Luo, X., Huang, X., and Gu, B. (2009) Influences of temperature on development and survival, reproduction and growth of a calanoid copepod (Pseudodiaptomus dubia). TheScientificWorldJOURNAL: TSW Environment 9, 866-879. DOI 10.1100/tsw.2009.96. 

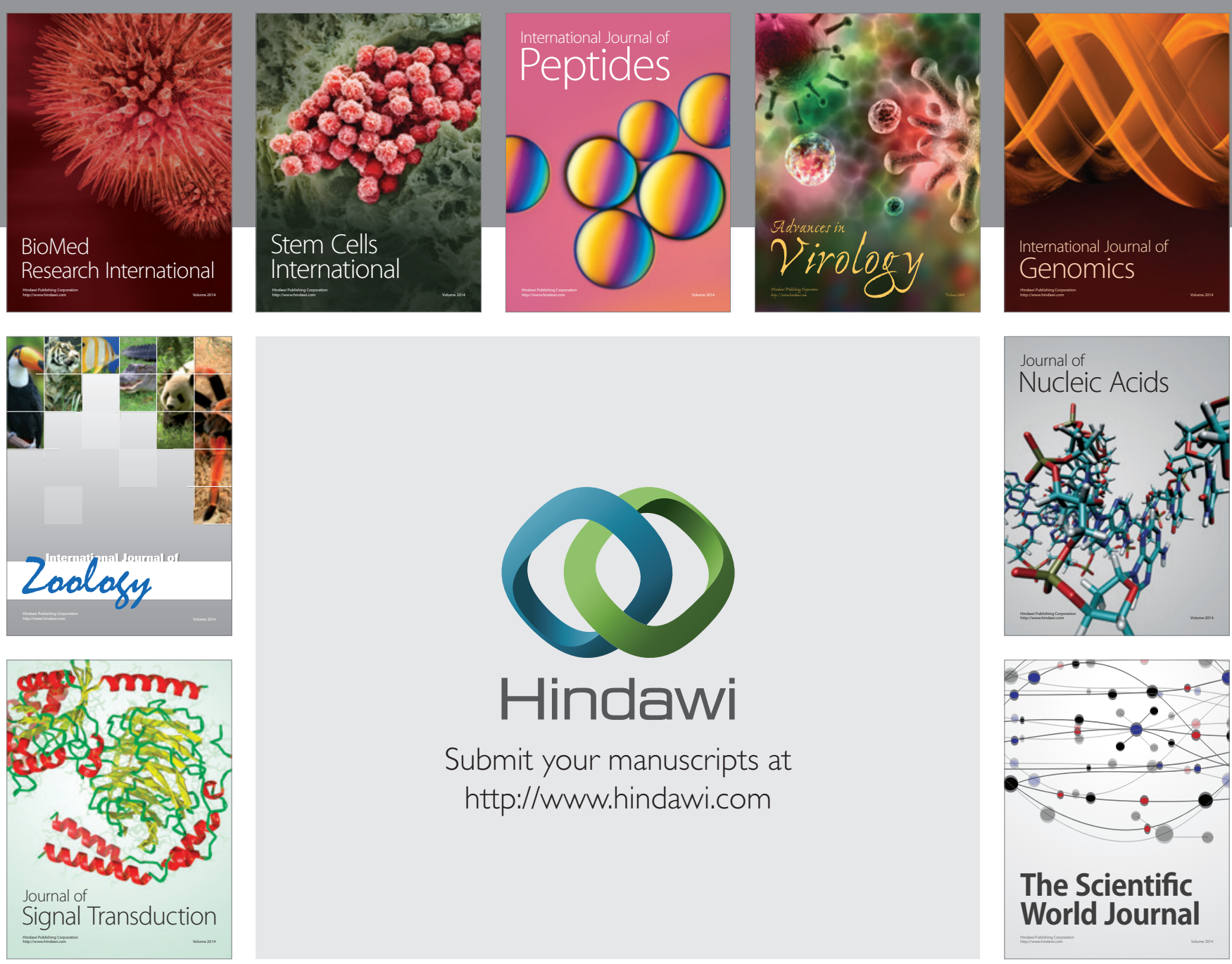

Submit your manuscripts at

http://www.hindawi.com
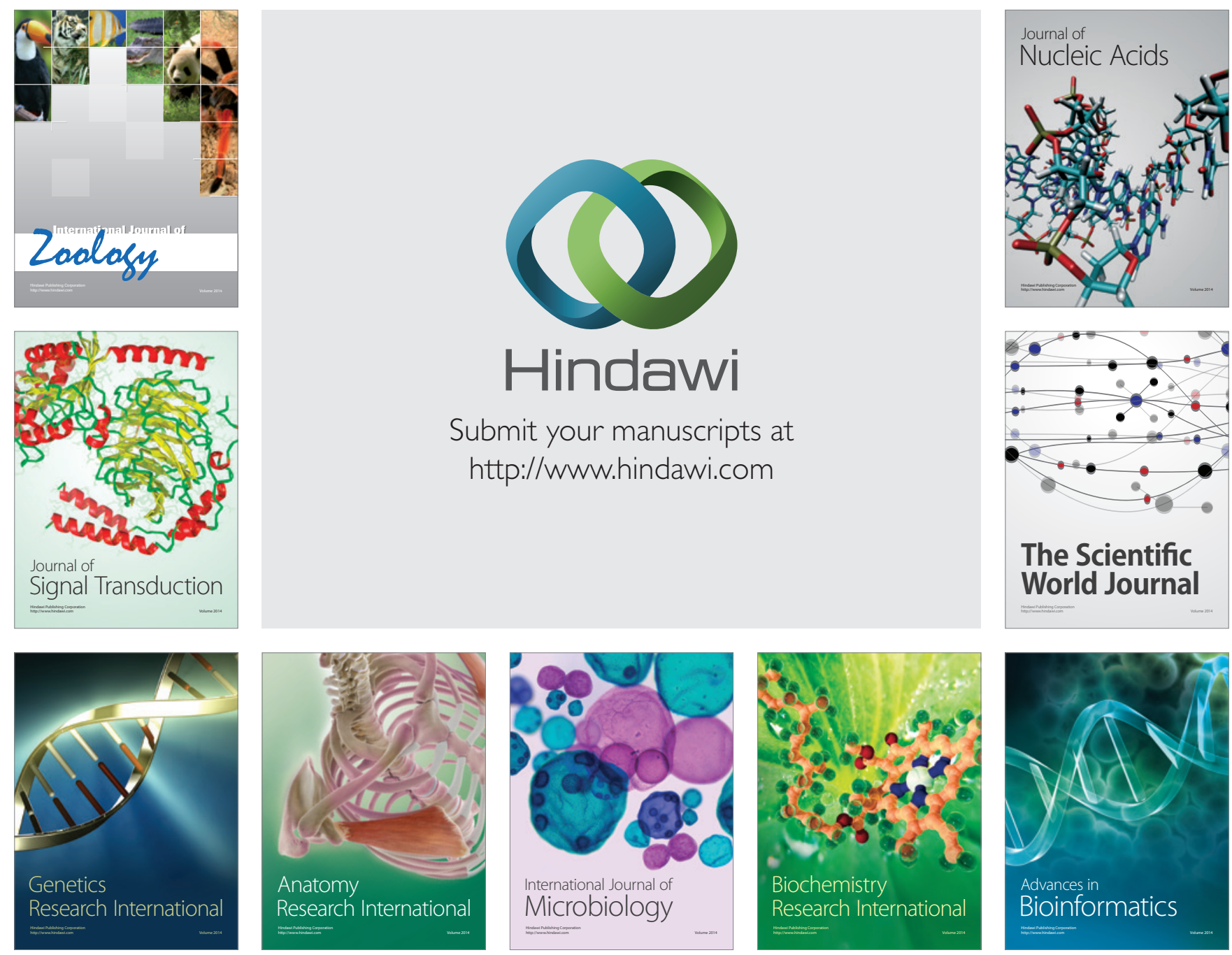

The Scientific World Journal
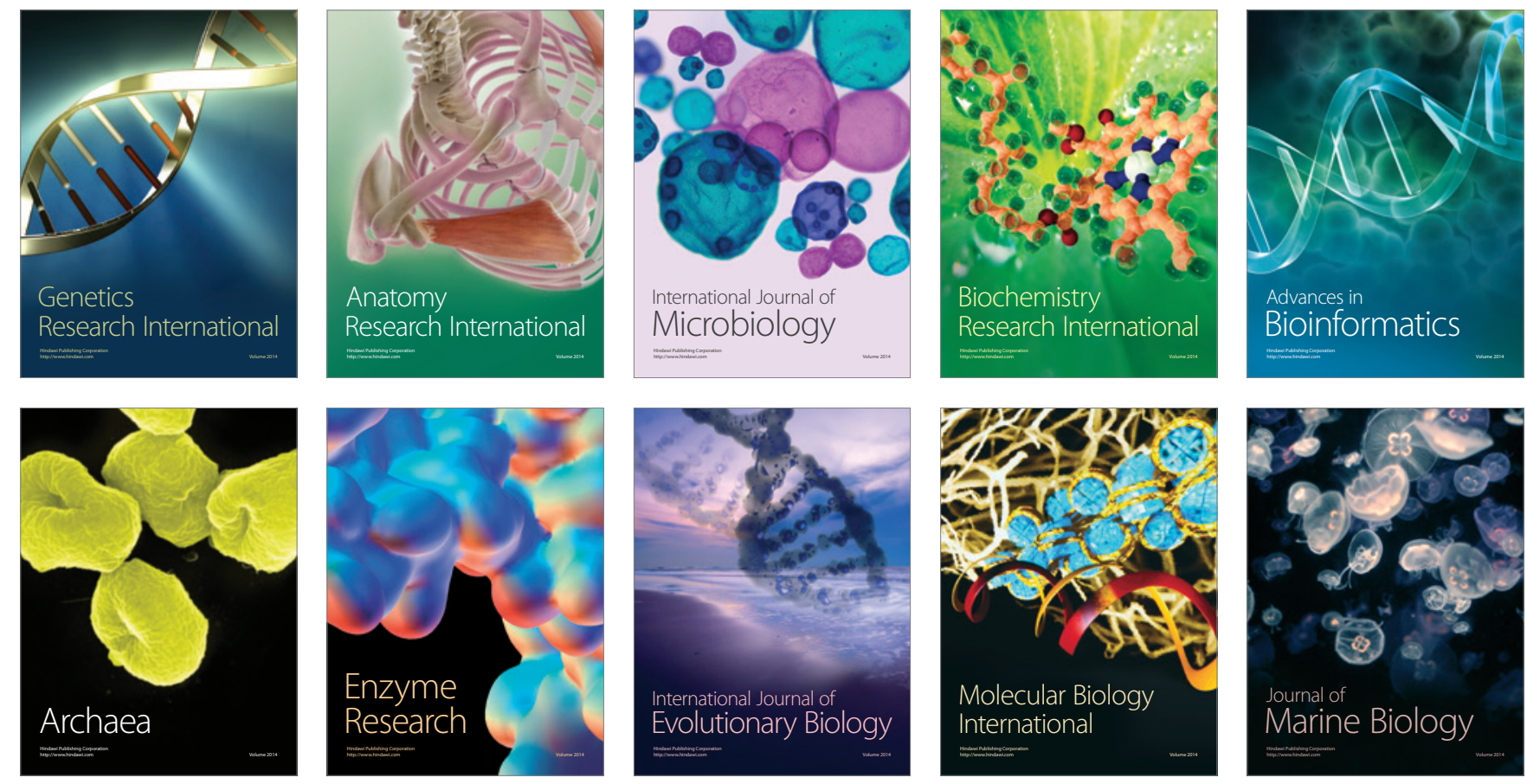